Supporting information

\title{
A Rationally Designed Supercharged Protein-enzyme Chimera Self-assembles in situ to Yield Bifunctional Composite Textiles
}

Graham J. Day', William H. Zhang ${ }^{1}$, Ben M. Carter ${ }^{1}$, Wenjin Xiao', Mark R. Sambrook ${ }^{2}$, and Adam W. Perriman ${ }^{1 *}$

${ }^{1}$ School of Cellular and Molecular Medicine, Biomedical Sciences Building, University of Bristol, University Walk, BS8 1TD, United Kingdom

${ }^{2}$ CBR Division, Defence Science and Technology Laboratory (Dstl), Porton Down, Salisbury, SP4 0JQ, United Kingdom

Corresponding author:

Adam W. Perriman

ORCID: 0000-0003-2205-9364

*Email: chawp@bristol.ac.uk 


\section{SUPPLEMENTARY MATERIALS AND METHODS}

Unless otherwise stated, all reagents were purchased from Sigma Aldrich, USA. All buffers and solutions were prepared in $18.2 \mathrm{M} \Omega \cdot \mathrm{cm}$ deionised water.

\subsection{Synthesis of scgfp-arpte}

The scgfp and arpte genes (Supplementary Table S1-4) were amplified, independently, by the polymerase chain reaction (PCR) using primers $a+c$ and $d+f$, respectively (Supplementary Table 1), using the Phusion Green Hot Start II High-Fidelity PCR Master Mix (Thermo Scientific, USA). Splicing-by-overlapping ends (SOE) ${ }^{1}$ was performed using PCR products of scgfp and arpte, with primers $a+f$ to produce scgfp-arpte. The pET-14b+ expression vector (Merck) was amplified by PCR using primers $g+h$ (Supplementary Table 1). The pET-14b+ PCR product and scgfp-arpte were double restriction digested with $\mathrm{Xhol}$ and $\mathrm{BamHI}$ prior to ligation of scgfp-arpte into dephosphorylated pET-14b+ PCR products with T4 ligase (New England Biolabs, USA). Subsequently, BL21(DE3) E. coli (New England Biolabs, USA) were transformed with pET14b+_scgfp-arpte. Unfused genes were also generated for protein expression.

\subsection{Sodium dodecyl sulphate polyacrylamide gel electrophoresis (SDS-PAGE)}

Protein fractions from IMAC or SEC were mixed in a 1:1 ratio with sample application buffer, which was comprised of, per 1 L: $300 \mathrm{~mL}$ glycerol, $500 \mathrm{~mL} 10 \%$ SDS, $100 \mathrm{~mL} 0.5 \mathrm{M}$ ethylenediaminetetraacetic acid (EDTA), $6 \mathrm{~g}$ Tris, adjusted to $\mathrm{pH} 7.0$ with hydrochloric acid, $50 \mathrm{~mL}$ $\beta$-mercaptoethanol, made up to $1 \mathrm{~L}$ with $\mathrm{dH}_{2} \mathrm{O}$. These mixtures were incubated at $95^{\circ} \mathrm{C}$ for 10 minutes then applied on to a Novex ${ }^{\mathrm{TM}} 4-20 \%$ Tris-Glycine gel (ThermoFisher Scientific, USA), submerged in running buffer (per $1 \mathrm{~L}: 2.9 \mathrm{~g}$ Tris-base, $14.4 \mathrm{~g}$ glycine, $1 \mathrm{~g}$ SDS, made up to $1 \mathrm{~L}$ with $\mathrm{dH}_{2} \mathrm{O}$ ) inside a Mini Gel Tank (ThermoFisher Scientific, USA) and a $225 \mathrm{~V}$ current was applied to the gel for 35 minutes. The gel was stained with Coomassie Blue ${ }^{\mathrm{TM}}$ stain and non-protein containing regions were de-stained with $\mathrm{dH}_{2} \mathrm{O}$ overnight to reveal the stained, denatured protein bands, which were compared to a protein molecular weight standard marker (PageRuler Plus, ThermoFisher Scientific, USA), run on the same gel.

\subsection{Matrix-assisted laser desorption/ionisation time-of-flight (MALDI-TOF) mass spectrometry}

Samples were diluted three-fold with $\mathrm{dH}_{2} \mathrm{O}$ and mixed in a 1:1 ratio with $\alpha$-cyano-4-hydroxycinnamic acid matrix (50\% acetonitrile, $0.1 \%$ trifluoracetic acid, $49.9 \% \mathrm{dH}_{2} \mathrm{O}$, and a-cyano-4hydroxycinnamic acid added until saturation). This was then spotted onto a ground-steel mass spectrometry plate until all the liquid had evaporated and loaded into an UltrafleXtreme MALDI-TOF 
mass spectrometer (Bruker Daltonics, USA) and the charge:mass ratio of the samples was measured in linear positive mode. The theoretical isotopically averaged mass was computationally determined using the ExPASy ProtParam tool using the given amino acid sequence. ${ }^{2}$

\subsection{UV-visible light spectroscopy}

The absorbance properties of protein samples were measured on Cary 60 UV-vis (Agilent Technologies). Samples were loaded into a $1 \mathrm{~cm}$ pathlength quartz cuvette and illuminated by a Xenon pulse lamp from 600-200 nm. The programme was baseline corrected with the buffer the protein sample was solubilised in.

\subsection{Bicinchoninic acid (BCA) protein concentration assay}

The micro-well plate $B C A$ assay was completed using the reagents and protocol from the Pierce BCA Protein Assay Kit (ThermoFisher Scientific, USA). Samples of bovine serum albumin (BSA) protein in the range concentration range $25-2000 \mu \mathrm{g} \cdot \mathrm{mL}^{-1}$ were prepared to generate a protein concentration standard curve at $\lambda=$ A562 using a Synergy Neo2 plate reader (BioTek). A line of linear regression was fitted to the standard curve and used to calculate the concentration of the sample protein.

\subsection{Molar extinction coefficients}

The Beer-Lambert law was used to determine the molar extinction coefficients at $280 \mathrm{~nm}\left(\varepsilon_{280}\right)$ and $487 \mathrm{~nm}\left(\varepsilon_{487}\right)$, for samples containing scGFP. The theoretical $\varepsilon_{280}$ was computationally determined using the ExPASy ProtParam tool using the given amino acid sequence.

\subsection{Full spectrum temperature unfolding and deconvolution}

Samples were prepared in CD buffer as before and were loaded into the CD spectrometer in a quartz cuvette. The spectrometer was programmed to collect single accumulations of data from $\lambda=$ 260-190 nm, with data pitch of $1 \mathrm{~nm}$, direct integration time (DIT) of $4 \mathrm{~s}$, a bandwidth of $2 \mathrm{~nm}$, a scanning speed of $5 \mathrm{~nm} \cdot \mathrm{min}^{-1}$, every $5^{\circ} \mathrm{C}$ from $25-95^{\circ} \mathrm{C}$. Individual temperature-dependent spectra were processed on the DichroWeb serve using the algorithms SELCON3, CONTIN/LL, and CDsstr. Raw data was provided along with all other necessary information from to process the data in MRE $[\theta]$. No optical scaling factors were used. Each algorithm utilised reference set 7 . The results from each programme were averaged and their standard deviations calculated.

\subsection{Polymer surfactant self-assembly experiments}

\subsubsection{Critical micelle concentration of surfactant of polymer surfactant}

$1 \mathrm{~mL}$ solutions of poly(ethylene glycol) 4-nonylphenyl 3-sulphopropyl ether ( $\left.\mathrm{S}^{-}\right)$were prepared in $\mathrm{dH}_{2} \mathrm{O}$ up to final concentrations $0-1000 \mu \mathrm{g} \cdot \mathrm{mL}^{-1}$, with $10 \mu \mathrm{L}$ 1,6-diphenyl-1,3,5-hexatriene solution 
(DPH; a saturated solution prepared by dissolving DPH in methanol). $200 \mu \mathrm{L}$ of each solution was pipetted into a 96 -well plate and equilibrated for $10 \mathrm{~min}$ on a rotator at $120 \mathrm{rpm}$. The fluorescence of the samples was measured (excitation at $\lambda=355 \mathrm{~nm}$ and emission at $\lambda=488 \mathrm{~nm}$ ) on a Mithras LB 940 plate reader (Berthold Technologies, Germany). Micelles were considered to be present when the fluorescence intensity was positive. A sigmoidal function was fitted to the data on Prism8 ${ }^{\mathrm{TM}}$ (GraphPad, USA).

\subsubsection{Critical micelle concentration vs sodium chloride concentration}

$1 \mathrm{~mL}$ aliquots of sodium chloride $(\mathrm{NaCl})$ solution were prepared over a concentration range of $0.1-$ $1000 \mathrm{mM}$, with $500 \mu \mathrm{g} \cdot \mathrm{mL}^{-1} \mathrm{~S}^{-}$and $10 \mu \mathrm{L} \mathrm{DPH}$ solution. $200 \mu \mathrm{L}$ of each solution was pipetted into a 96 -well plate and equilibrated for $10 \mathrm{~min}$ on a rotator at $120 \mathrm{rpm}$. The fluorescence of the samples was measured as above on a Neo2 plate reader (BioTek, USA).

\subsubsection{Critical micelle concentration during dialysis}

$500 \mu \mathrm{g} \cdot \mathrm{mL}^{-1}$ of $\mathrm{S}^{-}$solutions were incubated for one hour at room temperature with a magnet stirring at $200 \mathrm{rpm}$ to equilibrate. $5 \mathrm{~mL}$ of buffer ( $30 \mathrm{mM} \mathrm{HEPES}$ at pH 8.0 with $100 \mu \mathrm{M} \mathrm{CoCl}_{2}$ and $1 \mathrm{M} \mathrm{NaCl}$ ) was pipetted into a 14,000 MWCO semi-permeable membrane (BioDesign Dialysis Tubing ${ }^{\mathrm{TM}}$, ThermoFisher Scientific, USA) and sealed with clips. The semi-permeable membrane was then submerged in the dialysis buffer with a magnetic stirrer at $200 \mathrm{rpm}$. The concentration of $\mathrm{S}^{-}$inside and outside of the dialysis tubing was monitored for 24 hours by measuring the $A_{280}$ of $500 \mu \mathrm{L}$ on a Cary 60 UV-vis (Agilent Technologies, USA), from both inside and outside the semi-permeable membrane. The $500 \mu \mathrm{L}$ aliquot was replaced after measurement. The concentration of $\mathrm{S}^{-}$was determined from its experimentally derived extinction coefficient at $280 \mathrm{~nm}\left(\varepsilon_{280}: 949 \mathrm{M} \cdot \mathrm{cm}^{-1}\right)$, derived from the number average molecular weight of $\mathrm{S}^{-}, 1198 \mathrm{Da}(\mathrm{PDI}=1.03) .^{3}$ The number $(n)$ of $\mathrm{S}^{-}$molecules in solution was determined using the concentration $(c)$, the molecular weight, and Avogadro's constant ( $N_{a}$; Equation 1).

$$
n=\left(\frac{c}{\mathrm{MW}}\right) \times N_{a} \quad \text { Equation } 1
$$

\subsection{Zeta potentiometry}

Protein samples were syringe filtered through a $0.22 \mu \mathrm{m}$ filter, pipetted into a folded capillary Zeta cuvette, and loaded into a ZetaSizer Nano ZS (Malvern Panalytical, UK). The samples were incubated at $25{ }^{\circ} \mathrm{C}$ for 120 seconds. Data were collected from a minimum of 20 to a maximum of 200 measurement cycles. Nano-clusters were suspended in $\mathrm{dH}_{2} \mathrm{O}$ and measured as above. 


\subsection{Dynamic light scattering of [scGFP-arPTE][S-] nano-clusters}

[scGFP-arPTE][S ${ }^{-}$n nano-cluster in $\mathrm{dH}_{2} \mathrm{O}$ were centrifuged at $500 \mathrm{G}$ and the supernatant pipetted into a micro-volume disposable cuvette and loaded into a ZetaSizer Nano ZS (Malvern Panalytical, UK). The samples were incubated at $25^{\circ} \mathrm{C}$ for 300 seconds. Measurements were taken three times for each sample and averaged. The intensity data were plotted as frequency distributions and a Gaussian distribution function was plotted using the Prism ${ }^{\text {TM }} 8$ (GraphPad Software, USA).

\subsection{Bicinchoninic acid (BCA) assay of materials}

[scGFP-arPTE][S-] films in the wells of a 96-well plate were subjected to a BCA assay and [scGFParPTE][S-]-cotton textiles were adhered to the bases of a 24-well plate with $5 \mu \mathrm{L}$ of adhesive (Rapid Epoxy, Wilko, UK) to perform the assay. Protein concentrations were determined by fitting a line of linear regression to a BSA standard curve $\left(25-2000 \mu \mathrm{g} \cdot \mathrm{mL}^{-1}\right)$.

\subsection{Scanning electron microscopy}

Samples that were to be examined by scanning electron microscopy (SEM) were placed in a vacuum desiccator for at least 24 hours. They were then removed and mounted on to an aluminium specimen stub and sputter coated with a $15 \mathrm{~nm}$ gold/palladium layer. For high vacuum SEM imaging, samples were loaded into a FEI Quanta 200 field emission gun-SEM (ThermoFisher Scientific, USA). A beam energy of $8.0 \mathrm{kV}$ was used. The SEM equipment was accessed through the Wolfson Bioimaging Facility, Faculty of Life Sciences, University of Bristol.

\subsection{Synchrotron radiation-SAXS and WAXS}

SR-small- and wide-angle X-ray scattering (SAXS and WAXS) experiments were conducted on beamline 122 at Diamond Light Source, Didcot, UK. SAXS data is collected on a Pilatus P3-2M detector (Dectris, Switzerland), and WAXS data is collected on a Pilatus P3-2M-DLS-L detector (Dectris, Switzerland) at a fixed camera length. [scGFP-arPTE][S-] films were wetted with $\mathrm{dH}_{2} \mathrm{O}$ and placed between two pieces of Kapton film and clamped together in a differential scanning calorimetry pan. The pan was mounted in a quartz window on to the beam line for scattering. Data was processed on the Data Analysis WorkbeNch (DAWN) software package. ${ }^{4}$ Lorentz-correction of SAXS data was performed by converting the $I(q)$ vs $q$ axis to $I(q) \times q^{2}$ vs $q .{ }^{5}$

\subsection{Confocal microscopy}

All confocal microscopy was carried out at the Wolfson Bioimaging Facility, Faculty of Life Sciences, University of Bristol. Samples were placed into a $20 \mathrm{~mm}$ confocal dish (VWR®, USA) and mounted on to a Leica SP8 AOBS confocal laser scanning microscope attached to a Leica DMI6000 inverted epifluorescence microscope (Leica, UK). The excitation source used was an argon laser ( $\lambda=488$ 
Supplementary information

$\mathrm{nm})$. Three-dimensional stacked images were obtained using a $Z$-separation length of $5.0 \mu \mathrm{m}$. The images were captured using Leica LAS-X acquisition software (Leica, UK) and processed with Fiji software. 


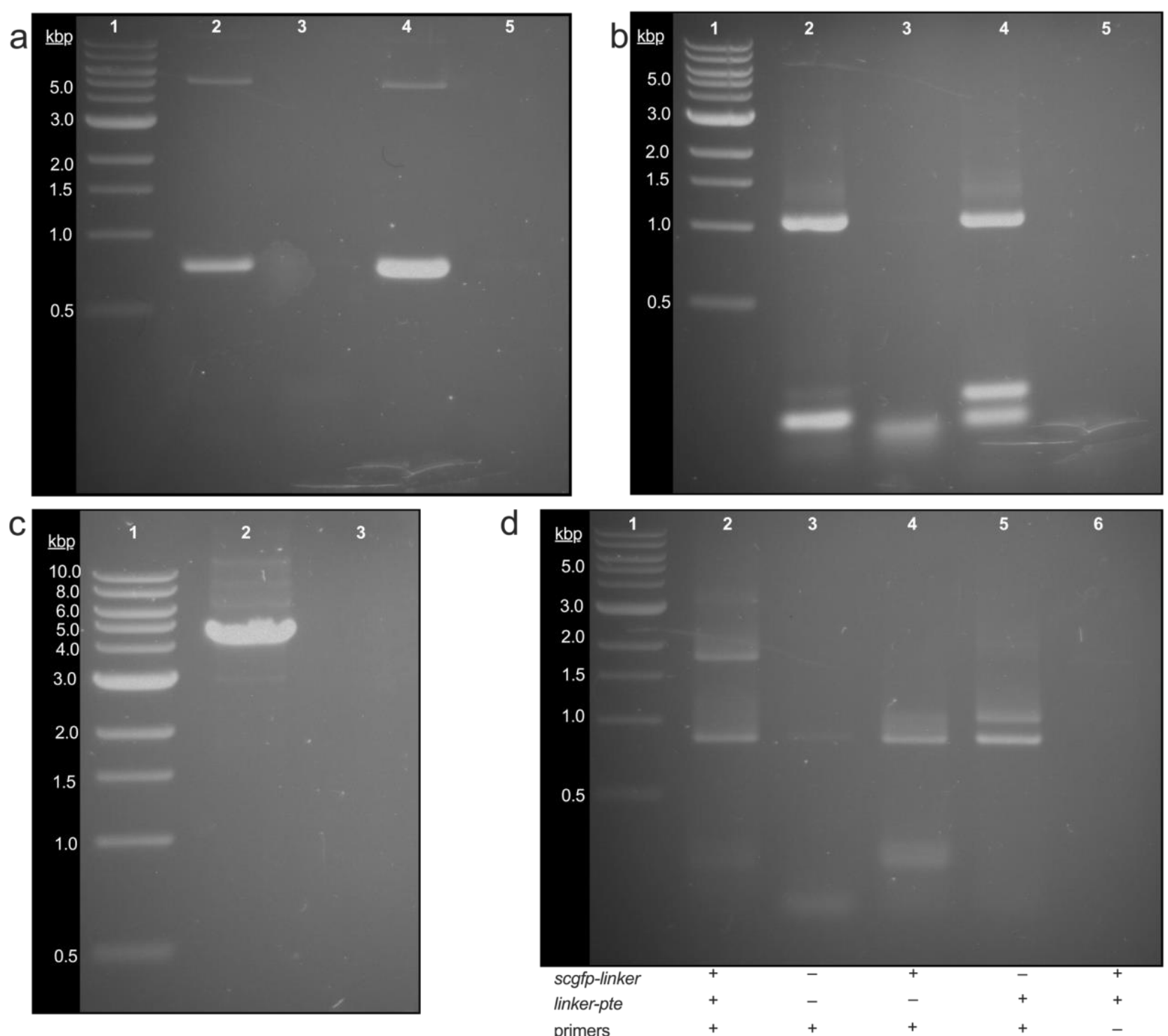

Figure S1. Agarose gel electrophoresis of amplified DNA for expression. a scgfp amplification products. Lane 1 contains a DNA size marker with select kilo-base pairs $(\mathrm{kbp})$ indicated. Lane 2 contains the reaction to generate scgfp-linker and lane 3 is this same reaction without template DNA. Lane 4 is the reaction to generate scgfp with no linker extension and lane 5 is this reaction without template DNA. The image was taken under UV-light $(\lambda=302 \mathrm{~nm})$ and exposed for $6 \mathrm{~s}$. b arpte amplification products. Lane 1 contains a DNA size marker with select kbp indicated. Lane 2 contains the reaction generate linker-arpte and lane 3 is this same reaction without template DNA. Lane 4 is the reaction to generate arpte with no linker extension, and lane 5 is this reaction without template DNA. The image was taken under UV-light $(\lambda=302$ $\mathrm{nm}$ ) and exposed for $6 \mathrm{~s}$. c $p E T-14 b$ amplification. Lane 1 contains a DNA size marker with select kbp indicated. Lane 2 contains the reaction to amplify $p E T-14 b$ and lane 3 is this same reaction without template DNA. The image was taken under UV-light $(\lambda=302 \mathrm{~nm}$ ) and exposed for $6 \mathrm{~s}$. d Splicing-by-overlapping-extension (SOE) reaction products. Below the gel, the presence or absence of the template DNA (scgfp-linker and linker-arpte) or the primers is indicated. Lane 1 contains a DNA size marker with select kbp indicated. Lane 2 contains the SOE reaction generate scgfp-arpte and lane 3 is this same reaction without template DNA. Lane 4 is the SOE reaction without linker-arpte, and lane 5 is the SOE reaction without scgfp-linker. Lane 6 is the SOE reaction without the forward or reverse primers. The image was taken under UV-light ( $\lambda=302 \mathrm{~nm}$ ), and exposed for $8 \mathrm{~s}$. 

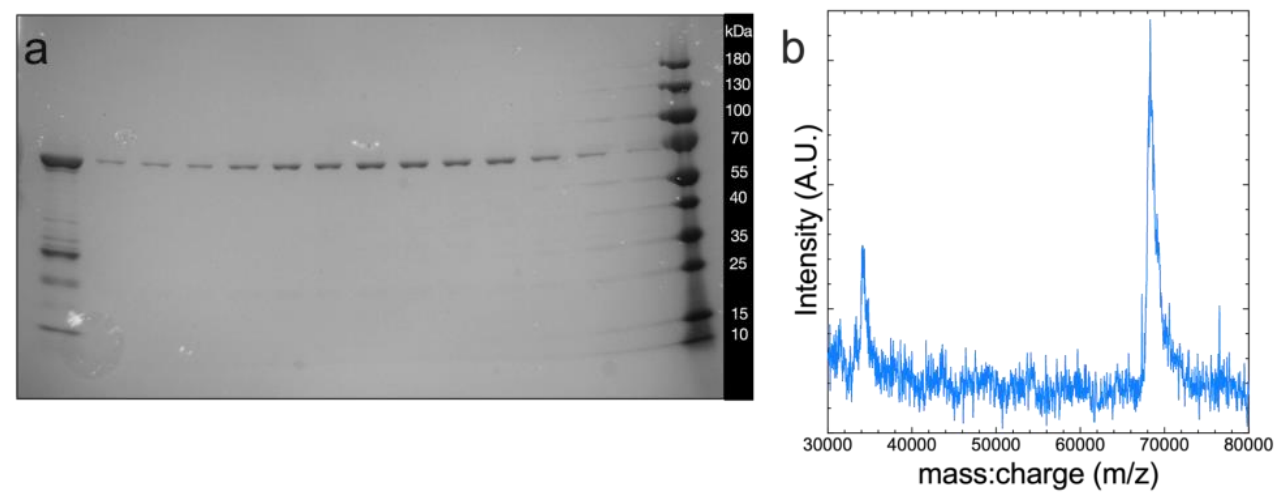

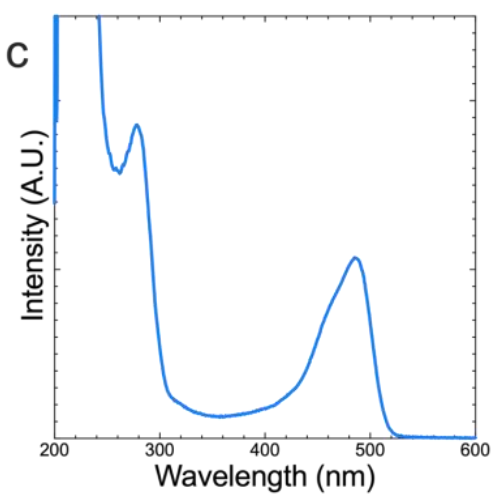

Figure S2. Purification of scGFP-arPTE. a Sodium dodecyl sulphate polyacrylamide gel electrophoresis (SDS-PAGE) of the eluted fractions from size exclusion chromatography (SEC) fractions. Lane 1 is the SEC load, lanes 2-13 correspond to elution fractions with highest $\mathrm{A} 280$ and A487 absorbance. Lane 15 is protein molecular weight (MW) marker. $\mathbf{b}$ Matrix-assisted laser desorption/ionisation time-of-flight (MALDI-TOF) mass spectrometry. Mass spectrum from MALDI-TOF analysis of the primary SEC peak. Samples in $30 \mathrm{mM}$ HEPES buffer at pH 8.0 with $100 \mu \mathrm{M} \mathrm{CoCl}_{2}$ and $1 \mathrm{M}$ sodium chloride was diluted 1/3 with deionised water then prepared in a 1:1 ratio with a-cyano-4hydroxycinnamic acid. c UV-vis spectrum of scGFP-PTE in undiluted buffer.
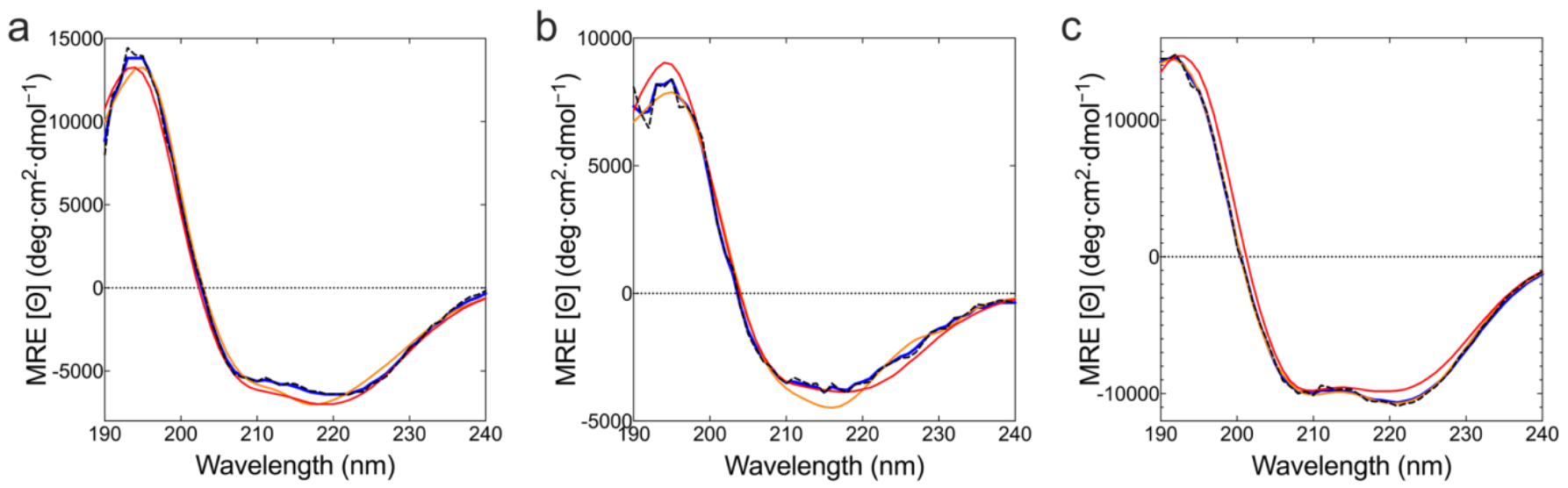

Figure S3. Fits to the circular dichroism spectra. a scGFP-arPTE, b scGFP, $\mathbf{c}$ arPTE. Raw data (black dotted lines) with SELCON3 (red), CONTIN (orange), and CDSSTR (blue) fits. 
a

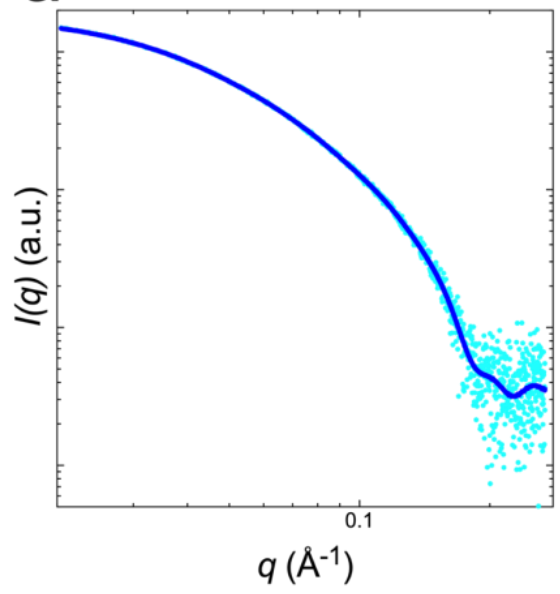

b

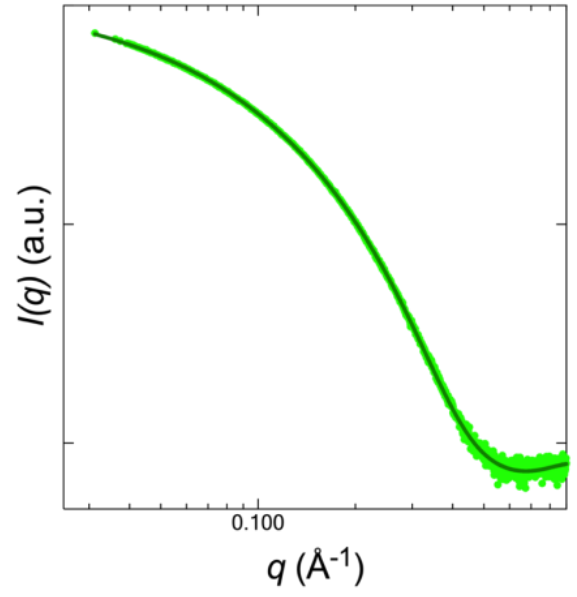

C

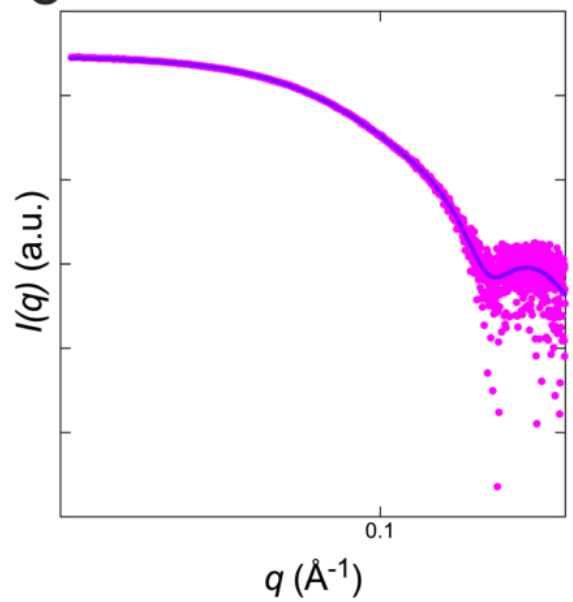

Figure S4. Synchrotron radiation-small angle X-ray scattering (SR-SAXS) studies. a Averaged intensity plots for scGFP-arPTE in $30 \mathrm{mM}$ HEPES buffer, $\mathrm{pH} 8.0$ with $1 \mathrm{M} \mathrm{NaCl}$. Chi squared $\left(X^{2}\right)$ for fit to the data is 1.00 . b Averaged intensity plot for scGFP in $20 \mathrm{mM}$ sodium phosphate buffer, $\mathrm{pH} 8.0$ with $1 \mathrm{M} \mathrm{NaCl}$. $X^{2}=0.81$. c Averaged intensity plot for arPTE in in $30 \mathrm{mM}$ HEPES buffer, $\mathrm{pH} 8.0$ with $1 \mathrm{M} \mathrm{NaCl} \chi^{2}=1.16$. The raw data is depicted as coloured circles with fits to the data overlaid as solid lines.
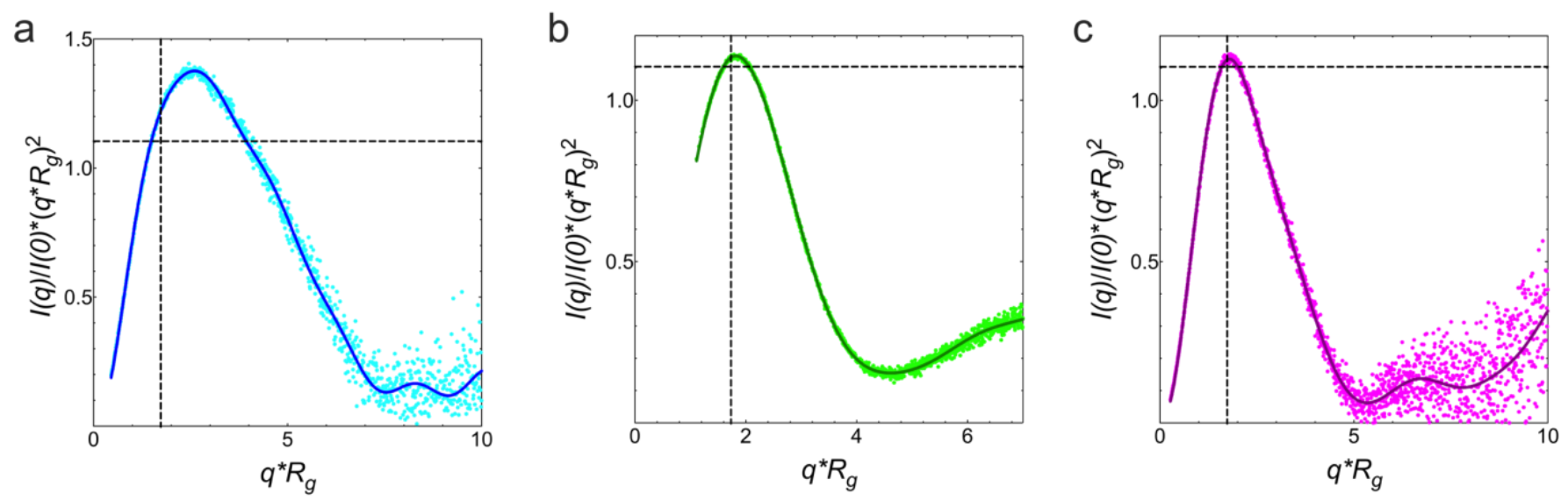

Figure S5. Dimensionless Kratky plots. a scGFP-arPTE small angle X-ray (SAXS) raw data (cyan circles) with fit (dark blue line). b scGFP SAXS raw data (light green circles) with fit (dark green line). c arPTE SAXS raw data (magenta circles) with fit (purple line). The intersection of the dashed lines indicates the coordinate $(\sqrt{3}, 1.104)$, the peak amplitude an ideal globular protein would exhibit on this plot. 


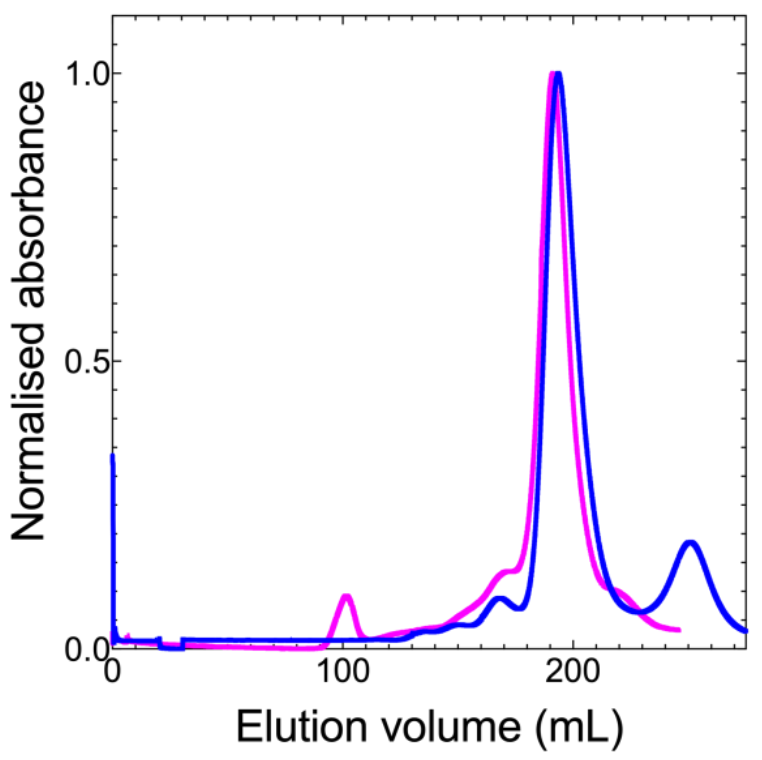

Figure S6. Size exclusion profile of scGFP-arPTE (blue line) and arPTE (magenta line). Absorbance was measured at $\lambda=280 \mathrm{~nm}$. The scGFP-arPTE was eluted in $30 \mathrm{mM}$ HEPES buffer at $\mathrm{pH} 8.0$ with $100 \mu \mathrm{M} \mathrm{CoCl} 2$ and $1 \mathrm{M}$ sodium chloride, whereas the arPTE was in $30 \mathrm{mM} \mathrm{HEPES}$ buffer at pH 8.0 with $100 \mu \mathrm{M} \mathrm{CoCl}_{2}$.

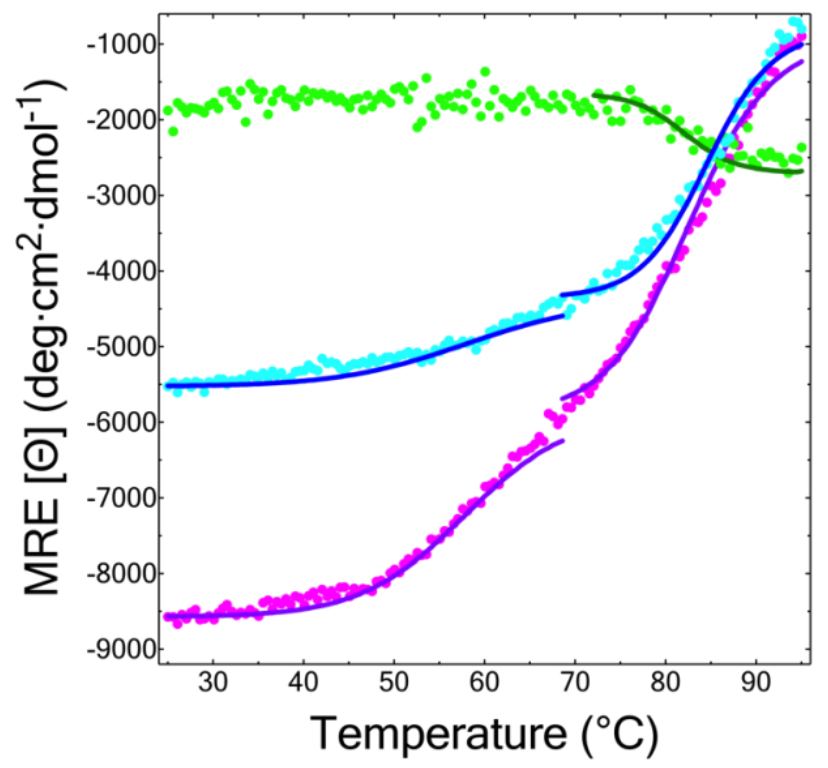

Figure S7. Thermal unfolding at $222 \mathrm{~nm}$. The circular dichroism of the scGFP-arPTE (blue), scGFP (green), and arPTE (magenta) was measured at $222 \mathrm{~nm}$ at increasing temperatures from $25-95{ }^{\circ} \mathrm{C}$, in $0.5{ }^{\circ} \mathrm{C}$ intervals. Equilibration time at each point was $12 \mathrm{~s}$. The raw data points are illustrated as coloured circles and fits to the data are overlaid as coloured solid lines. All measurements were carried out in $10 \mathrm{mM}$ potassium phosphate buffer at $\mathrm{pH}$ 8.0 , with $50 \mathrm{mM}$ sodium sulphate. 
a

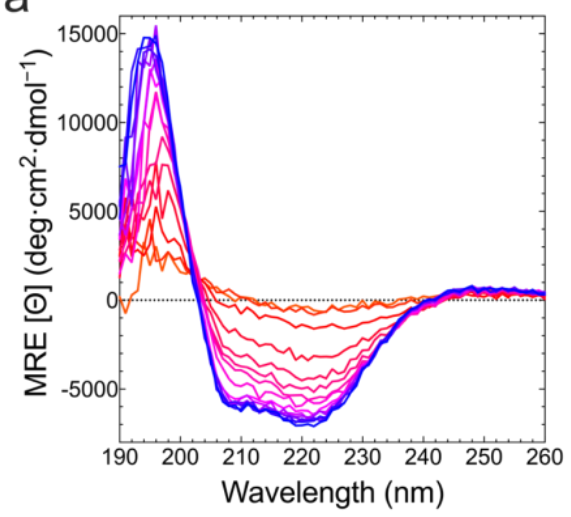

b

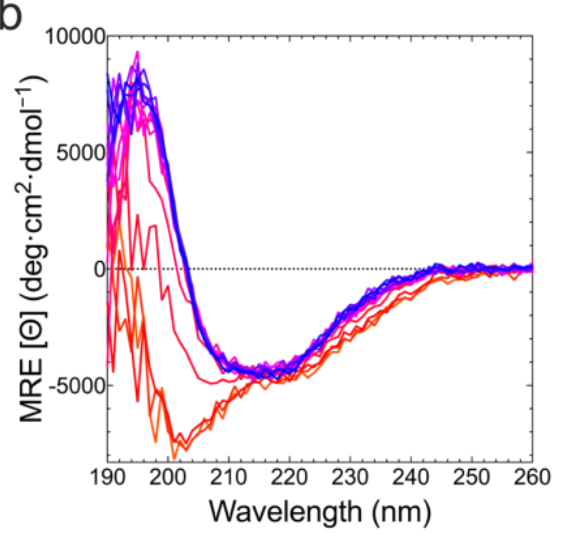

C

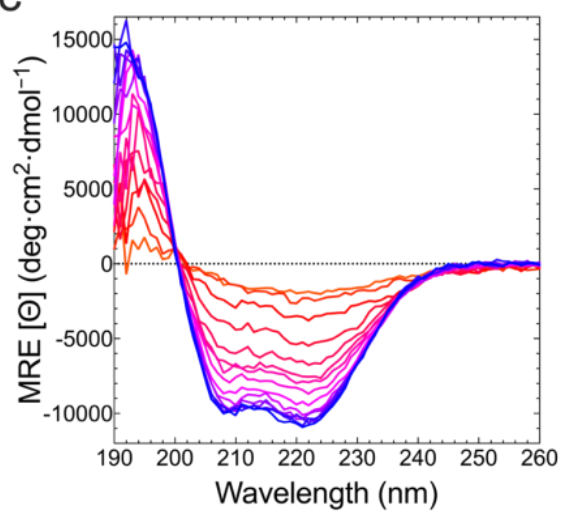

Figure S8. Circular dichroism (CD) spectra as a function of temperature. a scGFP-arPTE, b scGFP, and $\mathbf{c}$ arPTE. CD spectra were measured from at $25-95^{\circ} \mathrm{C}$ (blue through magenta to red) at $5{ }^{\circ} \mathrm{C}$ intervals. $\mathrm{CD}$ data were collected in 10 $\mathrm{mM}$ potassium phosphate buffer at $\mathrm{pH} 8.0$, with $50 \mathrm{mM}$ sodium sulphate. 

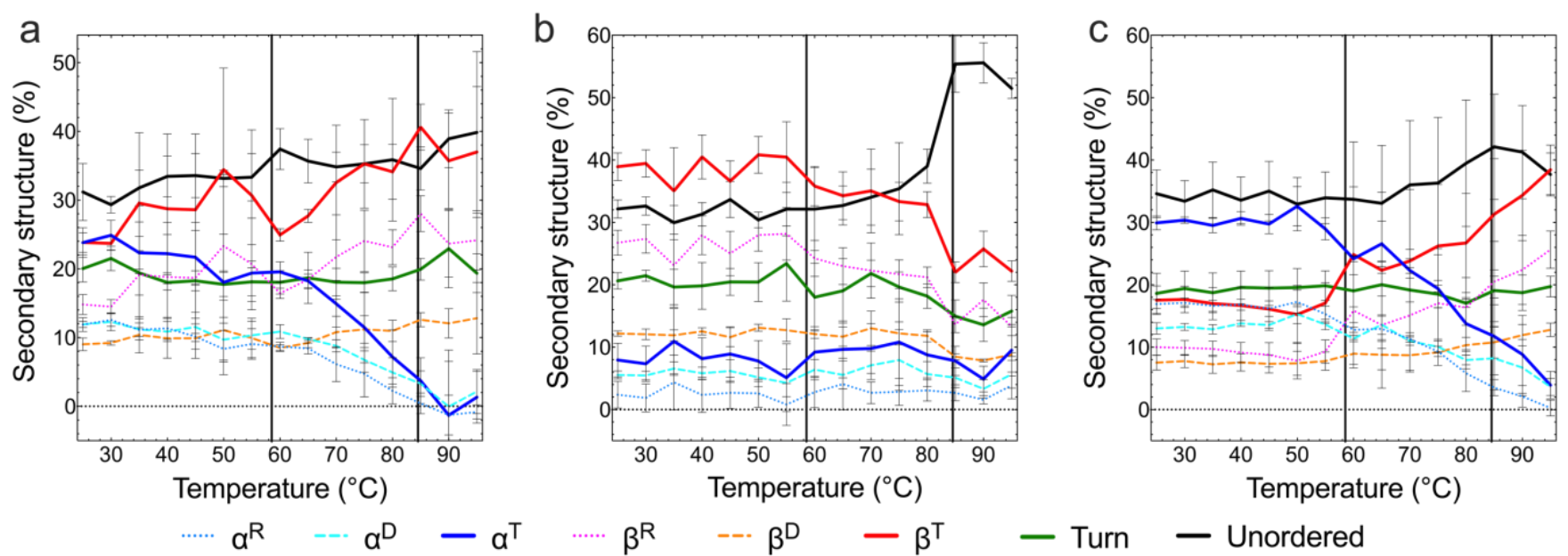

Figure S9. Deconvolution of temperature dependent circular dichroism spectra. a scGFP-arPTE, b scGFP, and c arPTE. The melting temperatures for each protein are indicated with vertical black lines. Regular $\alpha$-helix $\left(\alpha^{\mathrm{R}}\right)$, distorted $\alpha$-helix $\left(\alpha^{D}\right)$, total $\alpha$-helix $\left(\alpha^{\top}\right)$, regular $\beta$-sheets $\left(\beta^{R}\right)$, distorted $\beta$-sheets $\left(\beta^{D}\right)$, total $\beta$-sheet $\left(\beta^{\top}\right)$, turns, and unordered content are signified by the coloured lines (bottom). All data is expressed as an average of the results from the three deconvolution algorithms, and error bars are standard deviation.
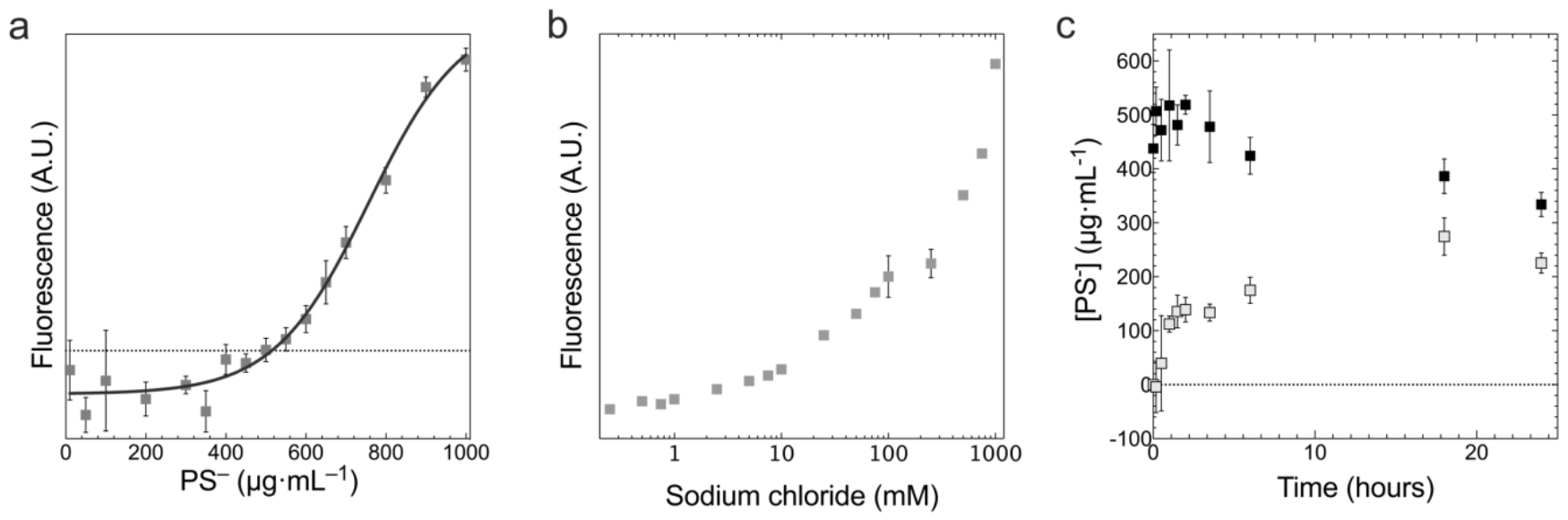

Figure S10. Modelling the critical micelle concentration $(C M C)$ of the anionic polymer surfactant, $\mathrm{S}^{-}$. a The fluorescence of 1,6-diphenyl-1,3,5-hexatriene (DPH) at $\lambda=488 \mathrm{~nm}$ in the presence of $\mathrm{S}^{-}$(grey squares), in $\mathrm{dH} 2 \mathrm{O}$. Results are an average with error bars as standard deviation $(n=3)$. A sigmoidal function was fitted (grey line; $R^{2}=0.96$ ). Micelles were considered to be present when the fluorescence was above 0 A.U. b The fluorescence of DPH at $\lambda=488 \mathrm{~nm}$ (grey squares) in $\mathrm{dH} 2 \mathrm{O}$ with $0.1-1000 \mathrm{mM}$ sodium chloride. Results are an average with error bars as standard deviation $(n=3)$. c Concentration of $\mathrm{S}^{-}$inside (grey squares) and outside (black squares) a semi-permeable dialysis membrane, over 24 hours. Initial inside conditions: $30 \mathrm{mM}$ HEPES at pH 8.0 with $100 \mu \mathrm{M} \mathrm{CoCl} 2$ and $1 \mathrm{M} \mathrm{NaCl}$. Initial outside conditions: $500 \mu \mathrm{g} \cdot \mathrm{mL}^{-1} \mathrm{~S}^{-}$in $\mathrm{dH} 2 \mathrm{O}$. Results are an average with error bars as standard deviation $(\mathrm{n}=3)$. All measurements were taken at $25^{\circ} \mathrm{C}$. 
Supplementary information

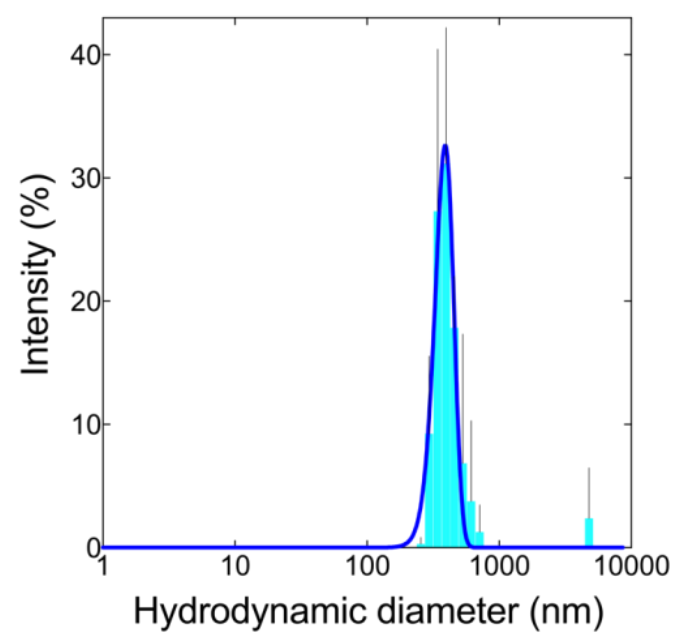

Figure S11. Dynamic light scattering studies after equilibrium dialysis. Intensity distributions of the [scGFParPTE][S-] nano-clusters (turquoise bars) suspension, with a Gaussian fit to the data (solid line). Data was collected three times and averaged. The sample was in deionised water and equilibrated for $180 \mathrm{~s}$ at $25^{\circ} \mathrm{C}$ before three measurements were taken. 


\section{Supplementary information}
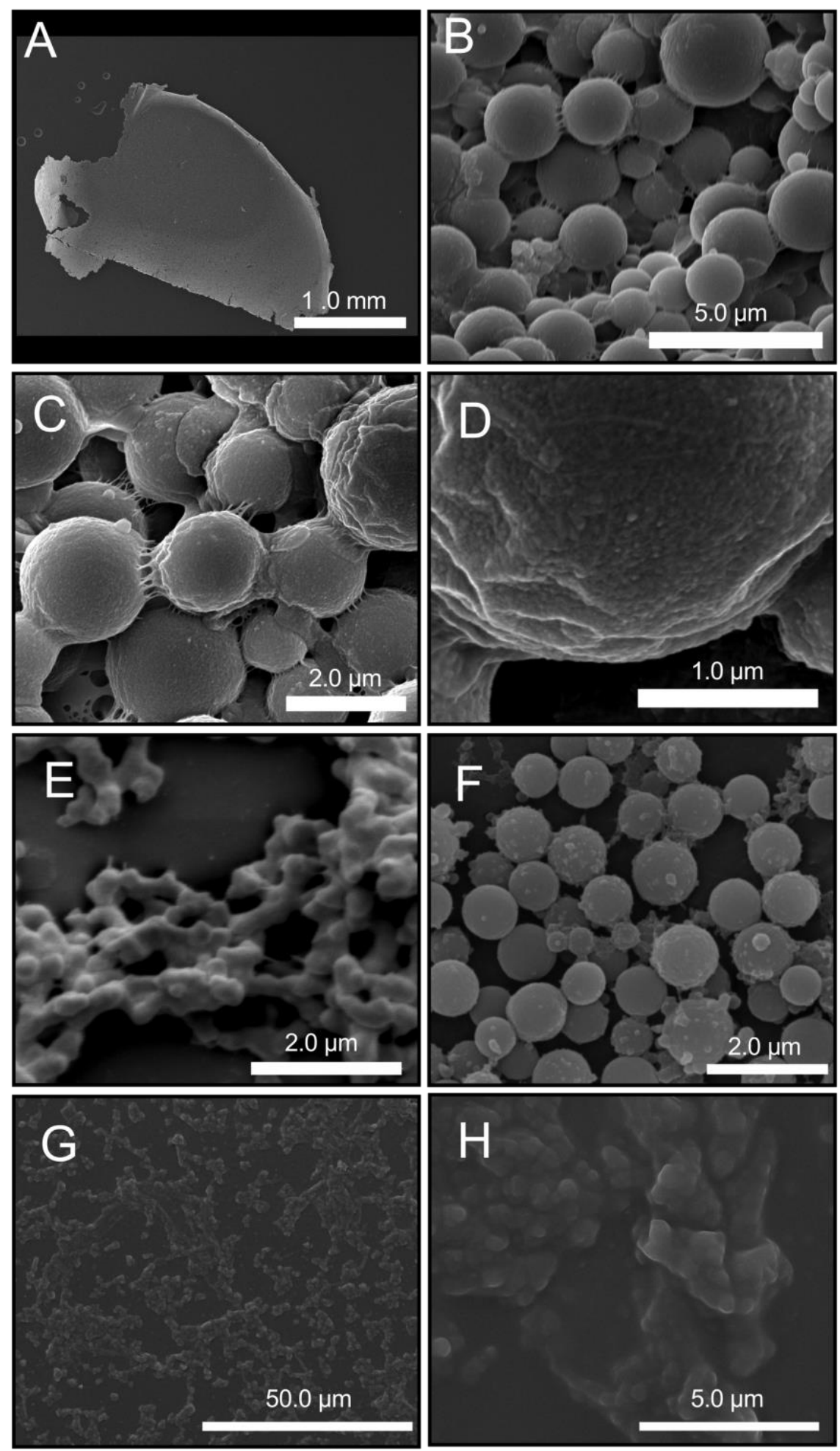

Figure S12. Scanning electron micrographs of cross-linked films. a Overview of the [scGFP-arPTE][S-] film at $\times 30$ magnification. $\mathbf{b}$ [scGFP-arPTE][S-] film imaged at $\times 25,000$ magnification. $\mathbf{c}$ [scGFP-arPTE][S-] film imaged at $\times 60,000$ magnification. d [scGFP-arPTE][S-] film imaged at $\times 80,000$ magnification. e scGFP-arPTE film with no polymer surfactant at $\times 20,000$ magnification. $\mathbf{f}$ [scGFP][S-] film imaged $\times 20,000$ magnification. $\mathbf{g}$ [arPTE][S-] film imaged at $\times 1,400$ magnification. $\mathbf{h}$ [arPTE][S-] film imaged at $\times 12,000$ magnification. All samples were coated with a $15 \mathrm{~nm}$ layer of $\mathrm{Au} / \mathrm{Pd}$ and imaged under high vacuum. The beam energy was $8.0 \mathrm{kV}$. 


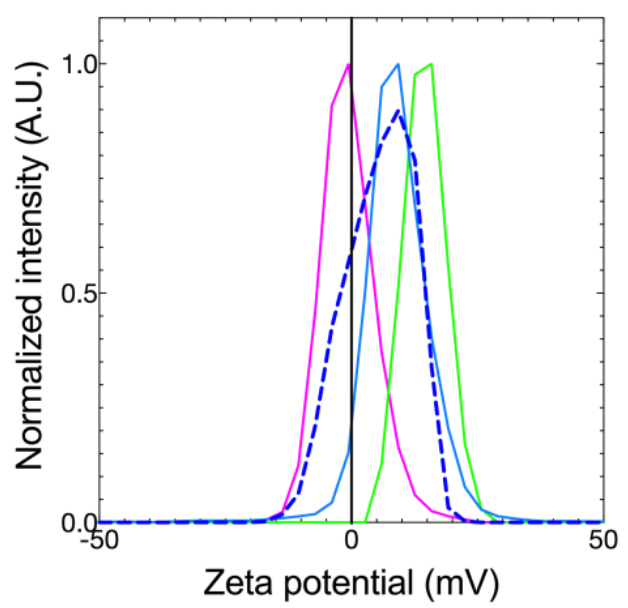

Figure S13. Zeta potentiometry. Relative intensity of zeta potential measurement for the aqueous scGFP-arPTE (light blue), scGFP (green), and arPTE (magenta) in 30 mM HEPES, pH 8.0. Also displayed is the zeta potential for the [scGFP-arPTE][S-] nano-clusters (dark blue dotted line), measurements of which were taken in pure water. Measurements were taken at $25^{\circ} \mathrm{C} . \mathrm{n}=6$ (scGFP-arPTE and arPTE), $\mathrm{n}=3$ (scGFP and scGFP-arPTE nanoclusters). A minimum of 20 and maximum 200 scans was enforced onto each measurement. Vertical black line is $0 \mathrm{mV}$.

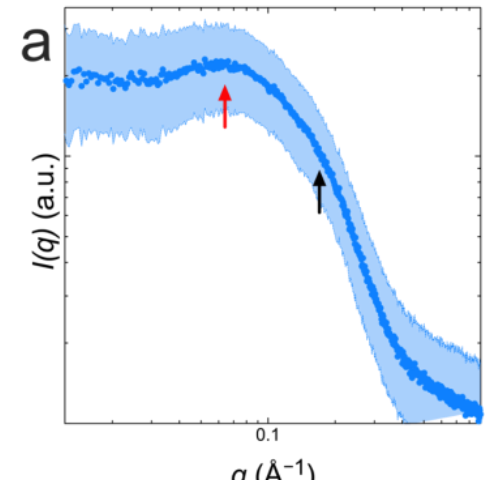

$$
q\left(\AA^{-1}\right)
$$
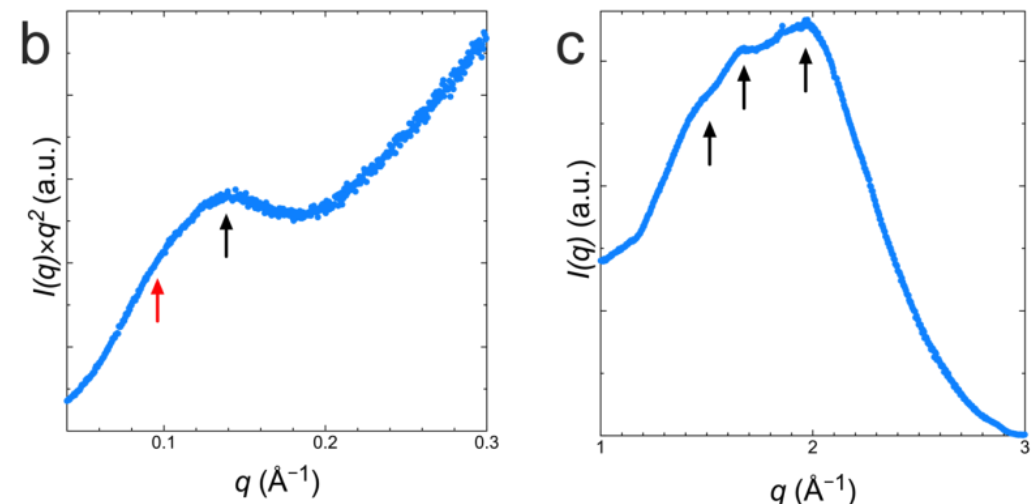

$q\left(\AA^{-1}\right)$

Figure S14. Small- and wide-angle X-ray scattering studies (SAXS and WAXS) of the [scGFP-arPTE][S-] film. a SAXS scattering data of the film. $\mathbf{b}$ Lorenz-corrected SAXS plot. Arrows indicate corresponding peaks in the data. c WAXS scattering data. The black arrows indicate peaks in the curve. The samples were wetted with deionised water at room temperature before the measurements were taken. 


\section{Supplementary information}

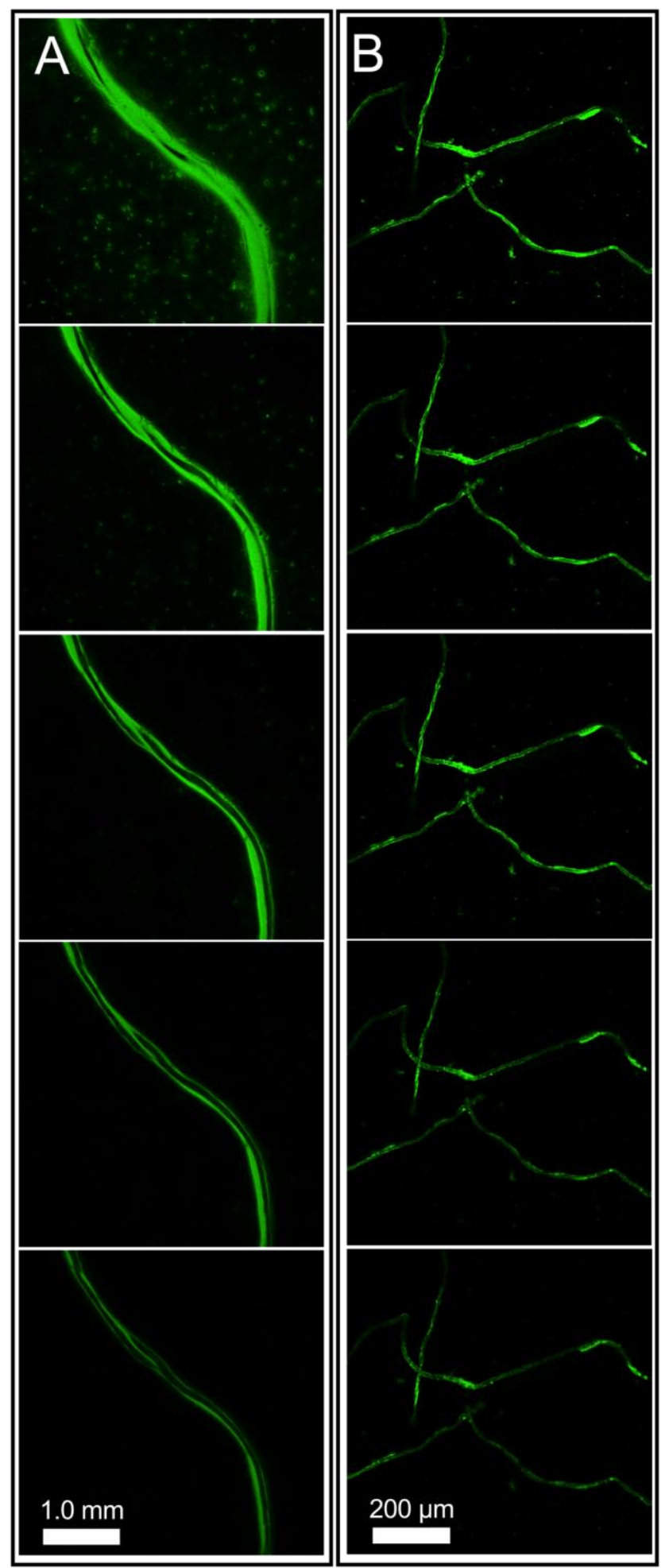

Figure S15. Confocal microscopy of cotton-fibre templated [scGFP-arPTE][S-] clusters. a A treated, woven cotton thread imaged through the $Z$-plane every $0.5 \mu \mathrm{m}$ with select images $25 \mu \mathrm{m}$ apart, at $\times 10$ magnification. The treated thread was illuminated under blue light $(\lambda=488 \mathrm{~nm})$ and imaged through a green light filter $(\lambda=510 \mathrm{~nm})$. b Unwoven, treated cotton fibres images through the $Z$-plane at $\times 40$ magnification under the same conditions as above. 


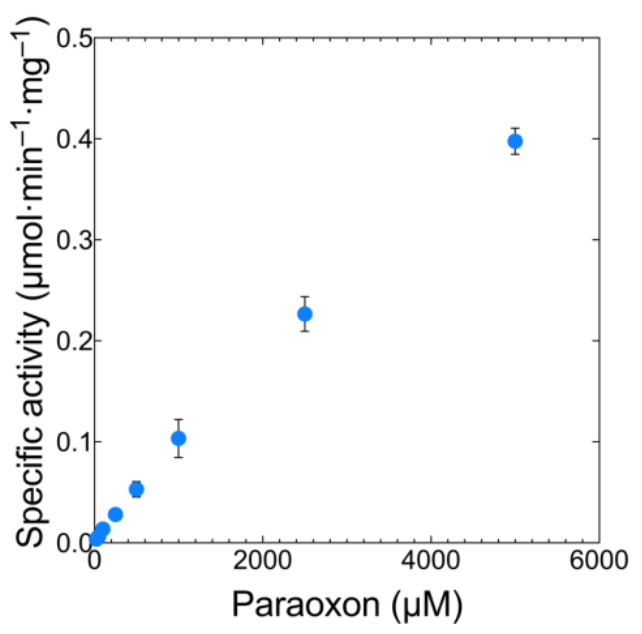

Figure S16. Michaelis-Menten plot of [scGFP-arPTE][S-]-cotton composites. Initial rates of paraoxon hydrolysis were averaged ( $n=3$, blue), error bars are standard All assays were conducted in $30 \mathrm{mM} \mathrm{HEPES}$ buffer at pH 8.0, containing $150 \mathrm{mM}$ sodium chloride and $100 \mu \mathrm{M}$ cobalt chloride at $25^{\circ} \mathrm{C}$.

Table S1. Primers used for the polymerase chain reaction and splicing-by-overlapping-extensions reaction. Forward primers corresponding to the genes are highlighted green (scGFP), purple (arPTE), or yellow (rigid linker). Reverse primers are highlighted black with the text in the same colours as described above. White text on black refers to the Xhol restriction digestion site, orange text on black corresponds to the reverse of the BamHI restriction digestion site, and blue indicates the STOP codon (TAG) added to the C-terminus of arPTE to facilitate translation termination. Black text on white are sequences corresponding to the plasmid insertion sites.

\begin{tabular}{|c|c|l|}
\hline \#primer & \multicolumn{1}{|c|}{ Primer name } & \multicolumn{1}{c|}{ Sequence } \\
\hline$a$ & Forward scgfp pET-14b & AGCCATATGCTCGAGGCTTCGAAAGGT \\
\hline$b$ & Reverse scgfp pET-14b & AGCCTCGAGCATATGGCTGCC \\
\hline$c$ & Reverse scgfp-linker & $\begin{array}{l}\text { ITTCGCCGCCGCTTCCTTTGCTGCTGCCTCG } \\
\text { CTGCCTTTATAGCGCTC }\end{array}$ \\
\hline$d$ & Forward arpte pET-14b & AGCCATATGCTCGAGACCGTGCGCGCGAGC \\
\hline$e$ & Reverse arpte pET-14b & ACGATTGCCGGATCCCTAGCTCGCGCGCACGGTCGG \\
\hline$f$ & Forward linker-arpte & $\begin{array}{l}\text { GAAGCGGCGGCGAAAGAGCTGCTGCTAAG } \\
\text { GGATCTATGAGCATGGCG }\end{array}$ \\
\hline$g$ & Forward $p E T-14 b$ & TAGGGATCCGGCTGCTAACAA \\
\hline$h$ & Reverse $p E T-14 b$ & AGCCTCGAGCATATGGCTGCC \\
\hline
\end{tabular}


Table S2. Nucleotide sequence for scGFP

GCT TCG AAA GGT GAA CGC CTG TTT CGC GGT AAA GTT CCG ATT CTT GTG GAA CTG AAA GGC
GAT GTG AAC GGA CAC AAA TTT TCG GTA CGT GGG AAA GGT AAA GGC GAT GCA ACC CGC
GGA AAA CTG ACC CTC AAA TTC ATT TGC ACC ACT GGC AAG CTG CCC GTT CCA TGG CCA ACC
TTA GTG ACA ACC TTG ACC TAT GGC GTT CAG TGC TTC TCT CGT TAT CCG AAA CAC ATG AAA
CGT CAT GAC TTC TTT AAA TCC GCG ATG CCG AAA GGC TAT GTG CAG GAA CGC ACG ATC AGC
TTT AAG AAA GAC GGC AAA TAC AAG ACT CGC GCG GAA GTC AAG TTT GAA GGA CGT ACG CTT
GTG AAT CGC ATC AAA CTG AAA GGG CGC GAT TTC AAA GAG AAA GGC AAC ATT CTG GGC CAC
AAA TTA CGG TAC AAC TTT AAC AGT CAC AAG GTC TAC ATT ACG GCC GAT AAA CGG AAG AAT
GGC ATC AAA GCG AAA TTC AAA ATT CGT CAC AAT GTG AAG GAT GGT AGC GTT CAA CTG GCC
GAT CAT TAC CAG CAG AAT ACG CCC ATT GGT CGT GGT CCG GTA TTG TTA CCG CGG AAC CAT
TAT CTG AGT ACA CGC TCA AAA CTG AGC AAA GAC CCG AAA GAA AAG CGC GAT CAT ATG GTA
CTG CTG GAA TTT GTC ACT GCA GCT GGG ATC AAG CAT GGT CGC GAT GAG CGC TAT

Table S3. Nucleotide sequence for arPTE.

ATG AGC ATG GCG CGC CCG ATT GGC ACC GGC GAT CTG ATT AAC ACC GTG CGC GGC CCG
ATT CCG GTG AGC GAA GCG GGC TTT ACC CTG ACC CAT GAA CAT ATT TGC GGC AGC AGC
GCG GGC TTT CTG CGC GCG TGG CCG GAA TTT TTT GGC AGC CGC AAA GCG CTG GTG GAA
AAA GCG GTG CGC GGC CTG CGC CAT GCG CGC GCG GCG GGC GTG CAG ACC ATT GTG GAT
GTG AGC ACC TTT GAT ATT GGC CGC GAT GTG CGC CTG CTG GCG GAA GTG AGC CGC GCG
GCG GAT GTG CAT ATT GTG GCG GCG ACC GGC CTG TGG TTT GAT CCG CCG CTG AGC ATG
CGC ATG CGC AGC GTG GAA GAA CTG ACC CAG TTT TTT CTG CGC GAA ATT CAG CAT GGC ATT
GAA GAT ACC GGC ATT CGC GCG GGC ATT ATT AAA GTG GCG ACC ACC GGC AAA GCG ACC
CCG TTT CAG GAA CTG GTG CTG CGC GCG GCG GCG CGC GCG AGC CTG GCG ACC GGC GTG
CCG GTG ACC ACC CAT ACC AGC GCG AGC CAG CGC GAT GGC GAA CAG CAG GCG GCG ATT
TTT GAA AGC GAA GGC CTG AGC CCG AGC CGC GTG TGC ATT GGC CAT AGC GAT GAT ACC
GAT GAT CTG AGC TAT CTG ACC GGC CTG GCG GCG CGC GGC TAT CTG GTG GGC CTG GAT
CGC ATG CCG TAT AGC GCG ATT GGC CTG GAA GGC AAC GCG AGC GCG CTG GCG CTG TTT
GGC ACC CGC AGC TGG CAG ACC CGC GCG CTG CTG ATT AAA GCG CTG ATT GAT CGC GGC
TAT AAA GAT CGC ATT CTG GTG AGC CAT GAT TGG CTG TTT GGC TTT AGC AGC TAT GTG ACC
AAC ATT ATG GAT GTG ATG GAT CGC ATT AAC CCG GAT GGC ATG GCG TTT GTG CCG CTG CGC
GTG ATT CCG TTT CTG CGC GAA AAA GGC GTG CCG CCG GAA ACC CTG GCG GGC GTG ACC
GTG GCG AAC CCG GCG CGC TTT CTG AGC CCG ACC GTG CGC GCG AGC

Table S4. Nucleotide sequence for the chimera scGFP-arPTE. The scGFP component is green, rigid linker is yellow, and arPTE is purple.

GCT TCG AAA GGT GAA CGC CTG TTT CGC GGT AAA GTT CCG ATT CTT GTG GAA CTG AAA GGC
GAT GTG AAC GGA CAC AAA TTT TCG GTA CGT GGG AAA GGT AAA GGC GAT GCA ACC CGC
GGA AAA CTG ACC CTC AAA TTC ATT TGC ACC ACT GGC AAG CTG CCC GTT CCA TGG CCA ACC
TTA GTG ACA ACC TTG ACC TAT GGC GTT CAG TGC TTC TCT CGT TAT CCG AAA CAC ATG AAA
CGT CAT GAC TTC TTT AAA TCC GCG ATG CCG AAA GGC TAT GTG CAG GAA CGC ACG ATC AGC
TTT AAG AAA GAC GGC AAA TAC AAG ACT CGC GCG GAA GTC AAG TTT GAA GGA CGT ACG CTT
GTG AAT CGC ATC AAA CTG AAA GGG CGC GAT TTC AAA GAG AAA GGC AAC ATT CTG GGC CAC
AAA TTA CGG TAC AAC TTT AAC AGT CAC AAG GTC TAC ATT ACG GCC GAT AAA CGG AAG AAT
GGC ATC AAA GCG AAA TTC AAA ATT CGT CAC AAT GTG AAG GAT GGT AGC GTT CAA CTG GCC
GAT CAT TAC CAG CAG AAT ACG CCC ATT GGT CGT GGT CCG GTA TTG TTA CCG CGG AAC CAT
TAT CTG AGT ACA CGC TCA AAA CTG AGC AAA GAC CCG AAA GAA AAG CGC GAT CAT ATG GTA




CTG CTG GAA TTT GTC ACT GCA GCT GGG ATC AAG CAT GGT CGC GAT GAG CGC TAT AAA GGC
AGC GAG GCA GCA GCA AAG GAA GCG GCG GCG AAA GAG GCT GCT GCT AAG GGA TCT ATG
AGC ATG GCG CGC CCG ATT GGC ACC GGC GAT CTG ATT AAC ACC GTG CGC GGC CCG ATT
CCG GTG AGC GAA GCG GGC TTT ACC CTG ACC CAT GAA CAT ATT TGC GGC AGC AGC GCG
GGC TTT CTG CGC GCG TGG CCG GAA TTT TTT GGC AGC CGC AAA GCG CTG GTG GAA AAA
GCG GTG CGC GGC CTG CGC CAT GCG CGC GCG GCG GGC GTG CAG ACC ATT GTG GAT GTG
AGC ACC TTT GAT ATT GGC CGC GAT GTG CGC CTG CTG GCG GAA GTG AGC CGC GCG GCG
GAT GTG CAT ATT GTG GCG GCG ACC GGC CTG TGG TTT GAT CCG CCG CTG AGC ATG CGC
ATG CGC AGC GTG GAA GAA CTG ACC CAG TTT TTT CTG CGC GAA ATT CAG CAT GGC ATT GAA
GAT ACC GGC ATT CGC GCG GGC ATT ATT AAA GTG GCG ACC ACC GGC AAA GCG ACC CCG
TTT CAG GAA CTG GTG CTG CGC GCG GCG GCG CGC GCG AGC CTG GCG ACC GGC GTG CCG
GTG ACC ACC CAT ACC AGC GCG AGC CAG CGC GAT GGC GAA CAG CAG GCG GCG ATT TTT
GAA AGC GAA GGC CTG AGC CCG AGC CGC GTG TGC ATT GGC CAT AGC GAT GAT ACC GAT
GAT CTG AGC TAT CTG ACC GGC CTG GCG GCG CGC GGC TAT CTG GTG GGC CTG GAT CGC
ATG CCG TAT AGC GCG ATT GGC CTG GAA GGC AAC GCG AGC GCG CTG GCG CTG TTT GGC
ACC CGC AGC TGG CAG ACC CGC GCG CTG CTG ATT AAA GCG CTG ATT GAT CGC GGC TAT
AAA GAT CGC ATT CTG GTG AGC CAT GAT TGG CTG TTT GGC TTT AGC AGC TAT GTG ACC AAC
ATT ATG GAT GTG ATG GAT CGC ATT AAC CCG GAT GGC ATG GCG TTT GTG CCG CTG CGC GTG
ATT CCG TTT CTG CGC GAA AAA GGC GTG CCG CCG GAA ACC CTG GCG GGC GTG ACC GTG
GCG AAC CCG GCG CGC TTT CTG AGC CCG ACC GTG CGC GCG AGC STOP

Table S5. Summary of the computationally and experimentally derived information of purified scGFP-arPTE.

\begin{tabular}{|cc|cc|c|}
\hline \multicolumn{2}{|c|}{ Molecular weight $(\mathrm{Da})$} & \multicolumn{2}{|c|}{$\varepsilon_{280}\left(\mathrm{M} \cdot \mathrm{cm}^{-1}\right)$} & $\varepsilon 487\left(\mathrm{M} \cdot \mathrm{cm}^{-1}\right)$ \\
\hline Estimated & Experimental & Estimated & Experimental & Experimental \\
67570 & 68318 & 46360 & $46495 \pm 1100$ & $34408 \pm 810$ \\
\hline
\end{tabular}




\section{Supplementary information}

Table S6. Deconvolution of the circular dichroism spectra. Values are the mean fraction (\%) of secondary structure from three algorithms. Error is standard deviation. $\alpha^{\mathrm{R}}$ is the regular $\alpha$-helix, $\alpha^{\mathrm{D}}$ is the distorted $\alpha$-helix, $\alpha^{\top}$ is total $\alpha$-helix content, $\beta^{R}$ is the regular $\beta$-sheet, $\beta^{D}$ is the distorted $\beta$-sheet, and $\beta^{T}$ is total $\beta$-sheet content.

\begin{tabular}{|c|c|c|c|c|c|c|c|c|}
\hline & $\alpha^{R}$ & $\alpha^{\mathrm{D}}$ & $\alpha^{\top}$ & $\beta^{R}$ & $\beta^{D}$ & $\beta^{\top}$ & Turns & Unordered \\
\hline scGFP-arPTE & $12 \pm 1$ & $10 \pm 1$ & $22 \pm 2$ & $17 \pm 1$ & $10 \pm 1$ & $26 \pm 1$ & $19 \pm 1$ & $32 \pm 2$ \\
\hline scGFP & $2 \pm 3$ & $6 \pm 1$ & $8 \pm 4$ & $28 \pm 3$ & $12 \pm 1$ & $40 \pm 3$ & $19 \pm 3$ & $31 \pm 1$ \\
\hline arPTE & $14 \pm 0$ & $11 \pm 1$ & $25 \pm 1$ & $10 \pm 2$ & $10 \pm 4$ & $19 \pm 1$ & $19 \pm 3$ & $35 \pm 6$ \\
\hline
\end{tabular}

Table S7. Structural parameters of aqueous proteins from the pair-distance distribution function derived from SR-SAXS experiments. $R_{g}$ is the radius of gyration, $D_{\max }$ is the maximum linear dimension, $r$ is the radial average inter-atomic distance, and $P_{E}$ is the Porod exponent.

\begin{tabular}{c|ccc}
\multicolumn{1}{c}{} & ScGFP-arPTE & scGFP & arPTE \\
\hline$R_{g}(\dot{A})$ & $41.5 \pm 4.7$ & $19.5 \pm 0.6$ & $27.4 \pm 0.3$ \\
$D_{\max }(\stackrel{\AA}{A})$ & 148.5 & 69.5 & 86.5 \\
Average $r(\AA)$ & 51.32 & 25.3 & 35.28 \\
$P_{E}$ & $3.17 \pm 0.04$ & $3.87 \pm 0.03$ & $3.64 \pm 0.03$
\end{tabular}

Table S8. Michaelis-Menten enzyme kinetic parameters of the scGFP-arPTE and arPTE. The $K_{m}$ is the Michaelis constant and $k_{c a t}$ is the turnover number. The values computed by applying a Michaelis-Menten fit to the initial rates of hydrolysis of paraoxon-ethyl.

\begin{tabular}{c|cc} 
& ScGFP-arPTE & arPTE \\
\hline$K_{m}(M)$ & $6.7 \times 10^{-5} \pm 1.4 \times 10^{-5}$ & $6.4 \times 10^{-6} \pm 2 \times 10^{-6}$ \\
$k_{\text {cat }}\left(\mathrm{s}^{-1}\right)$ & $16.83 \pm 0.82$ & $13.72 \pm 0.83$ \\
Specificity constant $\left(\mathrm{s}^{-1} \cdot \mathrm{M}^{-1}\right)$ & $2.5 \times 10^{5}$ & $2.2 \times 10^{6}$
\end{tabular}




\section{SUPPLEMENTARY REFERENCES}

(1) Heckman, K. L.; Pease, L. R. Gene Splicing and Mutagenesis by PCR-Driven Overlap Extension. Nat. Protoc. 2007, 2 (4), 924-932. https://doi.org/10.1038/nprot.2007.132.

(2) Gasteiger, E.; Hoogland, C.; Gattiker, A.; Duvaud, S.; Wilkins, M. R.; Appel, R. D.; Bairoch, A. Protein Analysis Tools on the ExPASy Server 571571 From: The Proteomics Protocols Handbook Protein Identification and Analysis Tools on the ExPASy Server.

(3) Gallat, F. X.; Brogan, A. P. S.; Fichou, Y.; McGrath, N.; Moulin, M.; Härtlein, M.; Combet, J.; Wuttke, J.; Mann, S.; Zaccai, G.; Jackson, C. J.; Perriman, A. W.; Weik, M. A Polymer Surfactant Corona Dynamically Replaces Water in Solvent-Free Protein Liquids and Ensures Macromolecular Flexibility and Activity. J. Am. Chem. Soc. 2012, 134 (32), 13168-13171. https://doi.org/10.1021/ja303894g.

(4) Basham, M.; Filik, J.; Wharmby, M. T.; Chang, P. C. Y.; El Kassaby, B.; Gerring, M.; Aishima, J.; Levik, K.; Pulford, B. C. A.; Sikharulidze, I.; Sneddon, D.; Webber, M.; Dhesi, S. S.; Maccherozzi, F.; Svensson, O.; Brockhauser, S.; Náray, G.; Ashton, A. W. Data Analysis WorkbeNch ( DAWN ). J. Synchrotron Radiat. 2015, 22 (3), 853-858. https://doi.org/10.1107/S1600577515002283.

(5) F. Cser. About the Lorentz Correction Used in the Interpretation of Small Angle X-Ray Scattering Data of Semicrystalline Polymers. J. Appl. Polym. Sci. 2001, 80 (12), 2300-2308. https://doi.org/10.1002/app.1335. 